\title{
Association of ApoE Gene Polymorphisms With Cardio- cerebrovascular Complications in Type 2 Diabetes Mellitus in the Chinese Population
}

\author{
Lulu Kong \\ First Affiliated Hospital of Soochow University \\ Yinting Gao \\ Jianhu County People' s Hospital \\ Wei Li \\ the Affiliated Hospital of Xuzhou Medical University \\ Bimin Shi ( $\square$ shibimin1987@163.com)
}

\section{Research article}

Keywords: ApoE gene polymorphism, Type 2 diabetes, Blood lipid, Cardio-cerebrovascular complications

Posted Date: July 15th, 2020

DOI: https://doi.org/10.21203/rs.3.rs-38669/v1

License: () (1) This work is licensed under a Creative Commons Attribution 4.0 International License. Read Full License 


\section{Abstract \\ Objective}

To analyze and study the relationship between ApoE gene polymorphism and cardio-cerebrovascular complications in type 2 diabetes mellitus(T2DM) in the Chinese Population.

\section{Methods}

From January 2018 to January 2019, 1140 patients with type 2 diabetes admitted to the Department of Endocrinology, the Affiliated Hospital of Xuzhou Medical University were selected as the case group, including 590 patients with coronary heart disease(CHD) and 550 patients with cerebral infarction(CI), and 1198 patients with type 2 diabetes without complications during the same period were selected as the control group. General baseline data of the two groups were collected, such as gender, age, course of disease, lipid profile, HbA1C, $\mathrm{BMI}$, blood pressure, carotid plaque and complications. ApoE genotypes were identified in all participants who participated in the study.

\section{Results}

This study showed that the ApoE genotypes in both the case group and the control group had the highest frequency of E3/E3. The E3/E4 genotype frequency and E4 allele frequency in the case group were higher than those in the control group $(P<0.05)$. In the case group, the frequency of E2/E3 and E3/E4 genotypes of $\mathrm{Cl}$ group was lower than that of $\mathrm{CHD}$ group, while the frequency of E3/E3 genotype was higher than that of CHD group. TC and LDL-c levels were significantly increased in patients with ApoE E3/E4 genotype $(P<0.05)$. ApoE genotype E3/E4 was more associated with carotid plaque than E2/E3. ApoE genotype and ApoE allele were positively correlated with TC and LDL-c levels $(P<0.05)$.Logistic regression results show that ApoE gene polymorphism is associated with cardio-cerebrovascular complications in T2DM patients. ApoE E3/E4 genotype and allele E4 may be risk factors for T2DM patients with cardio-cerebrovascular complications.

\section{Conclusion}

ApoE E3/E4 genotypes and T2DM patients carrying E4 allele have a higher risk of cardio-cerebrovascular complications than other genotypes. E4 allele may be a risk factor for cardio-cerebrovascular complications in T2DM patients, and its mechanism may be related to the effect of ApoE gene on lipid metabolism.

\section{Introduction}

The global incidence of T2DM has increased rapidly in recent years due to increased life expectancy in developing countries, rising rates of obesity and westernized lifestyles. However, chronic complications of T2DM are a major cause of morbidity and mortality [1,2]. Dyslipidemia or lipoprotein abnormality may aggravate microvascular and macrovascular complications in T2DM patients and promote atherosclerosis $[3,4]$. T2DM is a genetic disease related to plasma lipoprotein metabolism with obvious genetic predisposition and family cluster phenomenon. As an important component of plasma lipoprotein, apolipoprotein plays an extremely important role in the metabolism of plasma lipoprotein, so there is a close relationship between apolipoprotein and T2DM. Apolipoprotein $E$ (ApoE) is one of the important apolipoproteins involved in the metabolism of body lipids and the regulation of body lipids in human body. Its gene polymorphism is closely related to the level of body lipids [5]. This paper analyzed the ApoE genotype of T2DM patients with $\mathrm{Cl}$ and $\mathrm{CHD}$, and compared the blood lipid levels of patients with different ApoE phenotypes to explore the role of ApoE gene polymorphism in the occurrence and development of cardio-cerebrovascular complications of T2DM.

\section{Materials And Methods}

1.1 General information T2DM patients admitted to the Department of Endocrinology, the Affiliated Hospital of Xuzhou Medical University from January 2018 to January 2019 were collected. 1140 patients were selected as clinical subjects and set as case group, including 550 patients with CHD, including 280 males and 270 females, aged $47 \sim 81$ years, with an average age of (65.20 \pm 6.93$)$ years. There were 590 patients with $\mathrm{Cl}$, including 300 males and 290 females, aged $46 \sim 85$ years, with an average age of $(67.73 \pm 9.47)$ years. In addition, 1198 patients with T2DM without cardio-cerebrovascular complications in the same period were selected as the control group, including 600 males and 598 females, aged $44 \sim 85$ years, with an average age of $(64.71 \pm 7.26)$ years. There was no significant difference in gender

Page 2/8 
and age between the two groups $(P>0.05)$. General baseline data of the two groups were collected, such as course of disease, lipid profile, $\mathrm{HbA1C}, \mathrm{BMI}$, blood pressure, carotid plaque and complications. All subjects signed informed consent forms.

1.2 Inclusion criteria and Exclusion criteria The diagnosis of patients in the cerebral infarction group was in line with the standards of the Fourth National Conference on cerebrovascular diseases, which were confirmed by CT or MRI. According to the results of selective coronary angiography, at least two interventional physicians confirmed that the inner diameter of the main vascular lumen stenosis was $\geq 50 \%$. All patients in the case group and the control group were excluded from patients with two or more cardio-cerebrovascular diseases, severe liver and kidney diseases, blood diseases, severe infection, thyroid diseases and malignant tumors, and all subjects did not take lipid-lowering drugs.

1.3 Principles of ApoE genotyping detection Specific gene fragments amplified by PCR were hybridized with on-chip specific nucleic acid probes to detect specific gene locus sequences, including six ApoE gene types at sites 112 and 158 (E2/E2, E2/E3, E2/E4, E3/E3, E3/E4, E4/E4). The minimum detection limit of DNA was $5.0 \times 10^{3} \mathrm{copies} / \mathrm{ml}$, and $>5.0 \times 10^{3} \mathrm{copies} / \mathrm{ml}$ was positive for this site.

\subsection{Instruments and Reagents}

1.4.1 Determination of lipid profile and ApoE genotyping Fasting blood was collected early in the morning on the second day of admission for both groups. $2 \mathrm{ml}$ peripheral venous blood was collected with EDTA-K2 anticoagulant tube. After centrifugation, plasma was separated for ApoE genotyping.ApoE genotype detection kit (gene-chip assay) was purchased from Wuhan Youzhiyou Biotechnology Co., LTD., and the detection instrument was ABI7500 fluorescence quantitative PCR amplification instrument.Lipids include total cholesterol (TC), triglycerides (TG), high-density lipoprotein cholesterol (HDL-c), low-density lipoprotein cholesterol (LDL-c), apolipoprotein A (ApoA1), apolipoprotein B (ApoB). Beckman Coulter AU-5800 automatic biochemical analyzer was used for testing. All operations are strictly in accordance with the instructions.

1.4.2 Carotid ultrasound EPIQ5 ultrasonic diagnostic instrument was used with probe frequency of $1 \sim 18 \mathrm{MHz}$. Results and determination: carotid intimal thickness (IMT) was measured. Normal IMT: common carotid artery or its bifurcation IMT < 1.0 mm; Thickening of IMT: $1.0 \mathrm{~mm} \leq$ common carotid artery or its bifurcation IMT $<1.5 \mathrm{~mm}$; Plaque formation: IMT $\geq 1.5 \mathrm{~mm}$ in any carotid artery. The 2338 patients who underwent carotid ultrasound were divided into four groups: normal group, thickening group, stable plaque group and unstable plaque group. Stable plaque group: smooth surface, homogeneous or strong echo in more than $50 \%$ of plaque area, followed by sound shadow. Unstable plaque group: surface not smooth, $>50 \%$ plaque is heterogenous, low and irregular hypoechoic dark area or ulcerative plaque. If the subject has both stable and unstable plaques, they are classified as unstable plaques. All ultrasonic test results were determined by 2 professional ultrasound physicians.

1.5 Statistical methods The collected data and the clinical results have been statistically analyzed using IBM SPSS version 20.0 software. Quantitative data were expressed as mean values \pm standard deviation (SD). Ranges and frequency of distributions were estimated for quantitative variables. Normally distributed data were compared using Student's $t$ test for 2 groups and ANOVA test for more than 2 groups. The significance of differences between proportions was tested by the Chi square test $\left(\chi^{2}\right)$. Correlation analysis of ApoE genotype/allele and blood lipid level using Spearman rank correlation analysis. Differences were considered significant with $p$ value $<$ 0.05. Allele and genotype differences between groups and deviations from Hardy-Weinberg equilibrium were tested by Chi square test. Univariable logistic regression analysis was used to test the association between diseases and ApoE gene polymorphism and presented as unadjusted odds ratios (OR) with confidence interval $(95 \% \mathrm{Cl})$.

\section{Results}

2.1 General characteristics and biochemical variables of the study population

The course of diabetes, fasting blood glucose and $\mathrm{HbA1C}$ in the case group were significantly higher than those in the control group $(P<$ 0.05). The TC and LDL-c levels in the control group were lower than those in the case group, and the difference was statistically significant. The levels of TC and LDL-c in CHD group were higher than those in $\mathrm{Cl}$ group $(P<0.05)$.Demographic, clinical and biochemical data of enrolled subjects are summarized in Table 1.

\subsection{Results of ApoE gene polymorphism detection}

Six common ApoE genotypes E2/E2,E2/E3, E2/E4, E3/E3,E3/E4, E4/E4 and three alleles E2, E3 and E4 were detected by PCR-RFLP. Genotype E3/E3 was the most common (54.02\%), followed by E3/E4 (20.53\%), E2/E3 (19.63\%), and E2/E4 was the least common (0.77\%). E3 was the most common allele (74.10\%), followed by E4 (13.15\%) and E2 (12.75\%).

Page 3/8 
Through $\mathrm{H}-\mathrm{W}$ balance test, the distribution of ApoE genotype in the study subjects conforms to the law of genetic balance, indicating that the sample is representative $(P>0.05)$. The results showed that the ApoE genotypes in both the case group and the control group had the highest frequency of E3/E3, while the frequencies of the remaining genotypes in the case group were E3/E4, E2/E3 and E2/E4 from high to low, while the frequencies of the remaining genotypes in the control group were E2/E3, E3/E4 and E2/E4. The ApoE allele frequency in the case group was E3, E4 and E2 from high to low, while the control group was E3, E2 and E4. There was a significant difference between the ApoE genotype and allele frequency between the case group and the control group. The E3/E4 genotype of the case group was significantly higher than that of the control group $(P<0.05)$, while the E2/E3 genotype was significantly lower than that of the control group $(P<0.05)$. The E2 allele frequency in the case group was significantly lower than that in the control group $(P<0.05)$, while the $\mathrm{E} 4$ allele frequency was significantly higher than the control group $(P<0.05)$. In the case group, the frequency of E2/E3 and E3/E4 genotypes of $\mathrm{Cl}$ group was lower than that of CHD group, while the frequency of E3/E3 genotype was higher than that of CHD group. Comparison of ApoE genotype and allele frequency in each group is presented in Table 2.

2.4 Relationship between ApoE gene polymorphism and lipid metabolism

Comparison of blood lipid levels in patients with different ApoE genotypes showed that the levels of TC, LDL-c and ApoB in patients with E3/E4 genotype were significantly higher than those of E2/E3, E2/E4, E3/E3 genotypes $(P<0.05)$. The levels of TC, TG, LDL-C and ApoB in patients with E2/E4 and E3/E3 genotypes were higher than those with E2/E3 genotype $(P<0.05)$. Comparison of serum lipid levels among ApoE genotypes is presented in table 3 .

\subsection{Relationship between ApoE genotype and carotid atherosclerotic plaque}

Normal group and thickening group were classified as non-plaque group. Stable plaque group and unstable plaque group were classified as plaque group. The genotype frequency of ApoE E3/E4 in the plaque group was higher than that in the non-plaque group, while the frequency of E2/E3 and E2/E4 genotypes was lower than that in the non-plaque group $(P<0.05)$. Relationship between ApoE genotype and carotid atherosclerotic plaque is presented in table 4.

2.6 Correlation analysis of ApoE genotype, allele and blood lipid level

As shown in Table 5, ApoE genotype and ApoE allele were positively correlated with TC and LDL-c levels $(P<0.01)$.

2.7 Regression analysis between T2DM combined with cardio-cerebrovascular disease and ApoE gene polymorphism

Taking the occurrence of complications as the dependent variable, ApoE genotype or allele as the independent variable for Logistic regression, the results show that ApoE gene polymorphism is associated with the occurrence of cardio-cerebrovascular complications in T2DM patients .ApoE E3/E4 genotype $\left[X^{2}=13.32, P<0.01,95 \% \mathrm{Cl}(1.593 \sim 5.160)\right]$ and allele E4 $\left[\chi^{2}=9.31, P<0.01,95 \% \mathrm{Cl}(1.341 \sim 4.164)\right]$ might be the risk factors. The mechanism may be through the disorder of lipid metabolism and the promotion of lipid oxidation.

\section{Discussion}

Chronic complications of T2DM are the main cause of death and disability of diabetes mellitus, and macrovascular complications are the most common complications of T2DM. Diabetic macrovascular disease mainly refers to cardiovascular, cerebrovascular and peripheral vascular diseases. The main cause of cardio-cerebrovascular complications is atherosclerosis, which is one of the most common causes of human death. To date, some studies have shown that the interaction between T2DM and cardiovascular risk supports the progressive development of vascular injury, which leads to atherosclerosis [6]. Studies have pointed out that ApoE genotype is the main influencing factor for the development of atherosclerosis [7, 8], and differences in ApoE genotype can lead to differences in individual pathogenesis. Therefore, the study on ApoE genotype polymorphism has been paid more and more attention.

ApoE is involved in the regulation of lipid metabolism in the body through various ways, and is an important internal factor affecting the level of body lipid. The most common alleles of ApoE gene are E2, E3, E4, and there are 6 different ApoE phenotypes in the population: E2/E2, E2/E3, E2/E4, E3/E3, E3/E4, E4/E4, among which E3/E3 is the most common phenotype [9]. Studies at home and abroad have shown that ApoE gene polymorphism conform to the laws of genetics, but there are certain ethnic and regional differences, and there are differences in susceptibility to cardio-cerebrovascular diseases among ApoE individuals with different genotypes [10, 11]. In this study, the ApoE genotype of patients with T2DM and patients with cardio-cerebrovascular complications in the Affiliated Hospital of Xuzhou Medical University were statistically analyzed. The results showed that the ApoE genotypes in both the case group and the control group had the 
highest frequency of E3/E3. The frequencies of other genotypes in the case group were E3/E4, E2/E3 and E2/E4 from high to low, while those in the control group were E2/E3, E3/E4 and E2/E4. The comparison results between the case group and the control group showed that although the E3 allele frequency was the highest in both groups, the E2 and E4 allele frequency was significantly different, which showed that the E4 allele frequency in the case group was significantly higher than that in the control group, suggesting that E4 allele might be a risk factor for cardio-cerebrovascular diseases. The case group was divided into two types: coronary heart disease and cerebral infarction. ApoE is one of the important parameters for the occurrence of cardiovascular diseases. Studies have suggested that ApoE E4 allele is an independent risk factor for T2DM and coronary heart disease [12], but the correlation between ApoE and cerebral infarction is controversial. Some studies have pointed out that ApoE E4 allele is the genetic marker of cerebral infarction[13, 14], and other studies have suggested that ApoE E3/E3 and E3/E4 genotypes have protective effects on cerebral infarction in Chinese males rather than females [15]. In fact, ApoE gene polymorphism can affect lipid metabolism in a variety of ways, thus promoting or delaying the occurrence of cardiocerebrovascular diseases. In order to further clarify the ApoE gene polymorphism may play a role in cardio-cerebrovascular disease, blood lipid levels in patients with different phenotypes of ApoE this study are compared. The results showed that the TC and LDL-c levels of patients with ApoE E2 allele were significantly reduced, while the TC and LDL-c levels of patients with ApoE E4 allele were significantly increased, suggesting that in this study, ApoE E2 allele has a certain protective effect, and ApoE E4 allele may increase the incidence of cardio-cerebrovascular complications, which is basically consistent with the results of previous studies[16].

Atherosclerosis is an important risk factor for cardio-cerebrovascular diseases, and LDL-c is a key factor for the occurrence and development of atherosclerosis [17]. In this study, the comparison between the non-plaque and plaque groups showed that the ApoE E3/E4 genotype was more associated with carotid atherosclerotic plaque than E2/E3. This suggests that the correlation between ApoE and carotid atherosclerotic plaque may be caused by the influence of E3/E4 genotype on lipid LDL-c, which further leads to carotid atherosclerosis and plaque formation. It is consistent with other scholars research on ApoE E3/E4 genotype and carotid plaque[18, 19]. The results of this study show: E3/E4 genotype and E4 allele frequency of the case group were higher than of the control group. Logistic regression analysis of ApoE E3/E4 genotype and E4 allele is significantly associated with cardio-cerebrovascular complications. This suggests that the ApoE E3/E4 genotype and T2DM patients carrying E4 allele have a higher risk of cardio-cerebrovascular complications than other genotypes. E4 allele may be a risk factor for cardio-cerebrovascular complications in T2DM patients, and its mechanism may be related to the effect of ApoE gene on lipid metabolism.

This study shows that ApoE gene polymorphism does affect the lipid level, and ApoE E4 allele and E3/E4 genotype are significantly associated with the occurrence of carotid plaque and cardio-cerebrovascular complications, which has certain guiding significance for the early identification and prevention of the risk of complications in T2DM patients. ApoE polymorphisms seem to be very good candidates in studying the interplay between genetic and acquired risk factors. However, cardio-cerebrovascular diseases is the outcome of combined action of multiple factors. In the follow-up research, multi-center research should be carried out to increase the sample size of the research subjects for in-depth research. Future large-scale studies involving patients that will elucidate the pathophysiological pathways of cardiocerebrovascular complications may lead to new insights and treatments for diabetes.

\section{Abbreviations}

T2DM:Type 2 diabetes mellitus; Apolipoprotein E :ApoE; CHD :coronary heart disease; Cl: cerebral infarctio; IMT:intimal thickness;SBP: systolic blood pressure; DBP:diastolic blood pressure; HbA1c: hemoglobin A1C;TG:triglycerides; TC: total cholesterol; LDL-C: low density lipoprotein cholesterol; HDL-c:high density lipoprotein cholesterol; ApoA1: apolipoprotein A; ApoB:apolipoprotein B; BMl:body mass index.

\section{Declarations}

Acknowledgements

Not applicable.

Authors'contributions

LK and WL organized the study. YG participated in selection of patients. LK and YG made the genetic analysis, laboratory investigations and interpretation of data. BS made the statistical analysis. LK was a major contributor in writing the manuscript. BS revised the manuscript. All authors read and approved the final manuscript.

Funding 
The writing and revision of the manuscript was supported by the Department of Science \& Technology, Xuzhou, Jiangsu, China (Grant number: KC18213).

Availability of data and materials

The datasets used and analysed during the current study are available from the corresponding author on reasonable request.

Ethics approval and consent to participate

This study was conducted in accordance with the Declaration of Helsinki and approved by the Research Ethics Committee of the Affiliated Hospital of Xuzhou Medical University. Written informed consent was obtained from all participants.

Competing interests

The authors declare that they have no competing interests.

Author details

${ }^{1}$ Department of Endocrinology, The First Affiliated Hospital of Soochow University,Suzhou, Jiangsu, China

${ }^{2}$ Department of Endocrinology, The Affiliated Hospital of Xuzhou Medical University, Xuzhou, Jiangsu, China

${ }^{3}$ Department of Endocrinology, Jianhu County People's Hospital『Yancheng, Jiangsu, China

\section{References}

1. Guariguata L, Whiting DR, Hambleton I, Beagley J, Linnenkamp U, Shaw JE. Global estimates of diabetes prevalence for 2013 and projections for 2035. Diabetes Res Clin Pract. 2014;103(2):137-49.

2. Hossain P, Kawar B, El NM. Obesity and diabetes in the developing world-a growing challenge. N Engl J Med. 2007;356(3):213-5.

3. Mooradian AD. Dyslipidemia in type 2 diabetes mellitus. Nat Clin Pract Endocrinol Metab. 2009;5(3):150-9.

4. Jenkins AJ, Rowley KG, Lyons TJ, Best JD, Hill MA, Klein RL. Lipoproteins and diabetic microvascular complications. Curr Pharm Des. 2004;10(27):3395-418.

5. Skoog I. Vascular aspects in Alzheimer's disease. J Neural Transm Suppl. 2000;59:37-43.

6. Grundy SM. Pre-diabetes, metabolic syndrome, and cardiovascular risk. J AM COLL CARDIOL. 2012;59(7):635-43.

7. Toops KA, Tan LX, Lakkaraju A. Apolipoprotein E Isoforms and AMD. ADV EXP MED BIOL. 2016;854:3-9.

8. Ellulu MS, Patimah I, Khaza'Ai H, Rahmat A, Abed Y, Ali F. Atherosclerotic cardiovascular disease: a review of initiators and protective factors. INFLAMMOPHARMACOLOGY. 2016;24(1):1-10.

9. Mahley RW, Rall SJ. Apolipoprotein E: far more than a lipid transport protein. Annu Rev Genomics Hum Genet. 2000;1:507-37.

10. Velez-Pardo C, Rojas W, Jimenez-Del-Rio M, Bedoya G. Distribution of APOE polymorphism in the "Paisa" population from northwest Colombia (Antioquia). ANN HUM BIOL. 2015;42(2):195-8.

11. Kulminski AM, Arbeev KG, Culminskaya I, Ukraintseva SV, Stallard E, Province MA, Yashin Al. Trade-offs in the effects of the apolipoprotein E polymorphism on risks of diseases of the heart, cancer, and neurodegenerative disorders: insights on mechanisms from the Long Life Family Study. Rejuvenation Res. 2015;18(2):128-35.

12. Liu S, Liu J, Weng R, Gu X, Zhong Z. Apolipoprotein E gene polymorphism and the risk of cardiovascular disease and type 2 diabetes. BMC Cardiovasc Disord. 2019;19(1):213.

13. Dhungana H, Rolova T, Savchenko E, Wojciechowski S, Savolainen K, Ruotsalainen AK, Sullivan PM, Koistinaho J, Malm T. Westerntype diet modulates inflammatory responses and impairs functional outcome following permanent middle cerebral artery occlusion in aged mice expressing the human apolipoprotein E4 allele. J Neuroinflammation. 2013;10:102.

14. MacLeod MJ, De Lange RP, Breen G, Meiklejohn D, Lemmon H, Clair DS. Lack of association between apolipoprotein E genoype and ischaemic stroke in a Scottish population. EUR J CLIN INVEST. 2001;31(7):570-3.

15. Zhong Z, Wu H, Ye M, Yang Y, Luo W, Wu Y, Wu H, Zhong M, Zhao P. Association of APOE Gene Polymorphisms with Cerebral Infarction in the Chinese Population. Med Sci Monit. 2018;24:1171-7. 
16. Wang QY, Wang WJ, Wu L, Liu L, Han LZ. Meta-analysis of APOE $\varepsilon 2 / \varepsilon 3 / \varepsilon 4$ polymorphism and cerebral infarction. J Neural Transm (Vienna). 2013;120(10):1479-89.

17. Watkins H, Farrall M. Genetic susceptibility to coronary artery disease: from promise to progress. NAT REV GENET. $2006 ; 7(3): 163-73$.

18. Zhao LL, Su G, Chen LX, Yan Q, Wang XP, Yuan W, Wang L, Zhang ZC. Apolipoprotein E polymorphisms are associated with ischemic stroke susceptibility in a Northwest China Han population. Biosci Rep 2017, 37(6).

19. Shin MH, Choi JS, Rhee JA, Lee YH, Nam HS, Jeong SK, Park KS, Kim HY, Ryu SY, Choi SW, et al: APOE polymorphism and carotid atherosclerosis in Korean population: the Dong-gu Study and the Namwon Study. ATHEROSCLEROSIS 2014, 232(1):180-185.

\section{Tables}

Table 1

Demographic, clinical and biochemical data of the study population

\begin{tabular}{|c|c|c|c|}
\hline & CHD & $\mathrm{Cl}$ & Control \\
\hline Sex(male/female) & $280 / 270$ & $300 / 290$ & $600 / 598$ \\
\hline Age(years) & $65.20 \pm 6.93$ & $67.73 \pm 9.47$ & $64.71 \pm 7.26$ \\
\hline $\mathrm{SBP}(\mathrm{mmHg})$ & $129.95 \pm 14.18$ & $134.96 \pm 14.92$ & $129.07 \pm 14.68$ \\
\hline $\mathrm{DBP}(\mathrm{mmHg})$ & $79.50 \pm 9.96$ & $81.81 \pm 8.73$ & $81.35 \pm 7.75$ \\
\hline Diabetes duration(years) & $13.25 \pm 9.00$ & $12.59 \pm 8.18$ & $6.63 \pm 3.99^{\star} \Delta$ \\
\hline $\begin{array}{l}\text { Fasting plasma glucose } \\
(\mathrm{mmol} / \mathrm{L})\end{array}$ & $9.40 \pm 3.83$ & $8.60 \pm 2.67$ & $8.41 \pm 2.49^{\star} \Delta$ \\
\hline $\mathrm{HbA} 1 \mathrm{C}(\%)$ & $8.88 \pm 2.41$ & $8.83 \pm 2.16$ & $8.70 \pm 2.07^{\star \Delta}$ \\
\hline $\mathrm{TG}(\mathrm{mmol} / \mathrm{L})$ & $1.97 \pm 0.93$ & $2.14 \pm 1.01$ & $1.80 \pm 1.26$ \\
\hline $\mathrm{TC}(\mathrm{mmol} / \mathrm{L})$ & $5.16 \pm 1.42$ & $4.56 \pm 1.21^{*}$ & $3.51 \pm 1.40^{\star} \Delta$ \\
\hline LDL-c(mmol/L) & $3.04 \pm 1.11$ & $2.58 \pm 1.02^{*}$ & $2.30 \pm 1.00^{\star} \Delta$ \\
\hline $\mathrm{HDL}-\mathrm{c}(\mathrm{mmol} / \mathrm{L})$ & $1.18 \pm 0.29$ & $1.12 \pm 0.30$ & $1.20 \pm 0.35$ \\
\hline $\operatorname{ApoA1}(g / L)$ & $1.04 \pm 0.26$ & $1.02 \pm 0.19$ & $0.98 \pm 0.19$ \\
\hline $\operatorname{ApoB}(g / L)$ & $0.96 \pm 0.30$ & $0.83 \pm 0.25$ & $0.81 \pm 0.27$ \\
\hline $\mathrm{BMI}\left(\mathrm{kg} / \mathrm{m}^{2}\right)$ & $25.89 \pm 2.73$ & $25.45 \pm 2.74$ & $26.16 \pm 3.91$ \\
\hline
\end{tabular}

Table 2

Comparison of ApoE genotype and allele frequency in each group (\%)

\begin{tabular}{|c|c|c|c|c|c|c|c|c|c|c|}
\hline \multirow[t]{2}{*}{ Group } & \multirow[t]{2}{*}{$\mathrm{n}$} & \multicolumn{6}{|c|}{ Genotype frequency } & \multicolumn{3}{|c|}{ Allele frequency } \\
\hline & & E2/E2 & E2/E3 & E2/E4 & E3/E3 & E3/E4 & E4/E4 & E2 & E3 & E4 \\
\hline $\mathrm{CHD}$ & 550 & $\begin{array}{l}5 \\
(0.91 \%)\end{array}$ & $\begin{array}{l}94 \\
(17.09 \%)\end{array}$ & $\begin{array}{l}19 \\
(3.45 \%)\end{array}$ & $\begin{array}{l}262 \\
(47.63 \%)\end{array}$ & $\begin{array}{l}165 \\
(30.00 \%)\end{array}$ & $\begin{array}{l}5 \\
(0.91 \%)\end{array}$ & $\begin{array}{l}123 \\
(11.18 \%)\end{array}$ & $\begin{array}{l}783 \\
(71.18 \%)\end{array}$ & $\begin{array}{l}194 \\
(17.64 \%)\end{array}$ \\
\hline $\mathrm{Cl}$ & 590 & $\begin{array}{l}2 \\
(0.33 \%)\end{array}$ & $\begin{array}{l}47 \\
(7.96 \%)^{*}\end{array}$ & $\begin{array}{l}31 \\
(5.25 \%)\end{array}$ & $\begin{array}{l}378 \\
(64.07 \%)^{*}\end{array}$ & $\begin{array}{l}128 \\
(21.69 \%)^{*}\end{array}$ & $\begin{array}{l}4 \\
(0.68 \%)\end{array}$ & $\begin{array}{l}82 \\
(6.95 \%)^{*}\end{array}$ & $\begin{array}{l}931 \\
(78.90 \%)^{*}\end{array}$ & $\begin{array}{l}167 \\
(14.15 \%)^{*}\end{array}$ \\
\hline Control & 1198 & $\begin{array}{l}12 \\
(1.00 \%)\end{array}$ & $\begin{array}{l}318 \\
(26.54 \%)^{\star \Delta}\end{array}$ & $\begin{array}{l}49 \\
(4.09 \%)\end{array}$ & $\begin{array}{l}623 \\
(52.00 \%)^{\Delta}\end{array}$ & $\begin{array}{l}187 \\
(15.61 \%)^{\star \Delta}\end{array}$ & $\begin{array}{l}9 \\
(0.75 \%)\end{array}$ & $\begin{array}{l}391 \\
(16.32 \%)^{\star \Delta}\end{array}$ & $\begin{array}{l}1751 \\
(73.08 \%)^{\Delta}\end{array}$ & $\begin{array}{l}254 \\
(10.60 \%)^{\star} \Delta\end{array}$ \\
\hline
\end{tabular}


Table 3

Comparison of serum lipid levels among ApoE genotypes $(\overline{\mathrm{x}} \pm \mathrm{s})$

\begin{tabular}{|lllllll|}
\hline Genotype & $\begin{array}{l}\text { TC } \\
(\mathrm{mmol} / \mathrm{L})\end{array}$ & $\begin{array}{l}\text { TG } \\
(\mathrm{mmol} / \mathrm{L})\end{array}$ & $\begin{array}{l}\text { HDL-c } \\
(\mathrm{mmol} / \mathrm{L})\end{array}$ & $\begin{array}{l}\text { LDL-c } \\
(\mathrm{mmol} / \mathrm{L})\end{array}$ & $\begin{array}{l}\text { ApoA1 } \\
(\mathrm{g} / \mathrm{L})\end{array}$ & $\begin{array}{l}\text { ApoB } \\
(\mathrm{g} / \mathrm{L})\end{array}$ \\
\hline E2/E3 & $3.29 \pm 0.97$ & $0.86 \pm 0.32$ & $1.20 \pm 0.27$ & $1.60 \pm 0.66$ & $0.92 \pm 0.16$ & $0.58 \pm 0.13$ \\
\hline E2/E4 & $4.24 \pm 1.16^{*}$ & $2.14 \pm 0.80^{*}$ & $1.21 \pm 0.33$ & $2.36 \pm 0.94^{*}$ & $0.98 \pm 0.27$ & $0.78 \pm 0.25^{*}$ \\
\hline E3/E3 & $4.67 \pm 1.31^{*}$ & $1.84 \pm 1.64^{*}$ & $1.19 \pm 0.34$ & $2.66 \pm 1.04^{*}$ & $1.02 \pm 0.22$ & $0.86 \pm 0.30^{\star}$ \\
\hline E3/E4 & $5.64 \pm 1.31^{* \# \Delta}$ & $2.00 \pm 1.04$ & $1.17 \pm 0.29$ & $3.40 \pm 0.88^{* \# \Delta}$ & $1.06 \pm 0.22$ & $1.04 \pm 0.19^{\star \# \Delta}$ \\
\hline
\end{tabular}

Table 4

Relationship between ApoE genotype and carotid atherosclerotic plaque

\begin{tabular}{|lllll|}
\hline Carotid plaque & E2/E3 & E2/E4 & E3/E3 & E3/E4 \\
\hline Non- plaque group $(\mathrm{n}=684)$ & $\begin{array}{l}213 \\
(31.14 \%)\end{array}$ & $\begin{array}{l}57 \\
(8.33 \%)\end{array}$ & $\begin{array}{l}327 \\
(47.80 \%)\end{array}$ & $\begin{array}{l}87 \\
(12.72 \%)\end{array}$ \\
\hline $\begin{array}{l}\text { plaque group } \\
(\mathrm{n}=1654)\end{array}$ & 272 & 10 & 847 & 525 \\
$\mathrm{P}$ & $(16.44 \%)$ & $(0.60 \%)$ & $(51.21 \%)$ & $(31.74 \%)$ \\
\hline
\end{tabular}

Table 5

Correlation analysis of ApoE genotype, allele and blood lipid level

\begin{tabular}{|llcll|}
\hline blood lipid levels & \multicolumn{2}{l}{ ApoE genotype } & \multicolumn{2}{l|}{ ApoE allele } \\
\cline { 2 - 5 } & $\mathbf{r}$ & $\mathbf{P}$ & $\mathbf{r}$ & $\mathbf{P}$ \\
\hline TC & 0.360 & $<0.01$ & 0.332 & $<0.01$ \\
\hline TG & -0.024 & 0.732 & -0.054 & 0.433 \\
\hline HDL-C & -0.037 & 0.594 & -0.034 & 0.621 \\
\hline LDL-C & 0.360 & $<0.01$ & 0.333 & $<0.01$ \\
\hline
\end{tabular}

